\title{
Comparing the Absolute Power of Brain Waves in the Frontal Area among People with Major Depressive Disorder, Obsessive-Compulsive Disorder and Normal
}

\author{
Sajjad Basharpoor $^{1^{*}}$, Shirin Ahmadi ${ }^{1}$, Parviz Molavi², Fazeleh Heidari ${ }^{1}$
}

${ }^{1}$ School of Educational Sciences and Psychology, University of Mohaghegh Ardabili, Ardabil, Iran

${ }^{2}$ School of Medicine, Ardabil University of Medical science, Ardabil, Iran

Article Info:

\section{A BSTRACT}

Introduction: Attempting to recognize specific QEEG markers in depression and obsessive-compulsive disorders is the one of main interests of research in quantitative electroencephalography. The purpose of the present study was to compare the absolute power of brain waves in the frontal area in people with major depressive disorder and obsessive-compulsive disorder. Materials and Methods: The method of this study is causal-comparative. The statistical population of this study consisted of all individuals with major depressive disorder and obsessive-compulsive disorder referring to the mental health Clinic of Fatemi Hospital in 2019 in Ardabil, Iran. 15 people with major depressive disorder and 15 subjects with obsessive-compulsive disorder were selected by purposeful sampling. Furthermore, 15 normal individuals were selected via the sampling method from the relatives of patients. Psychiatric diagnosis and structured clinical interview, Beck depression inventory, and Foa et al. obsessive-compulsive inventory were used to collect data. The QEEG recording was performed at the Psychological Laboratory of Mohaghegh Ardabili University and the data were analyzed by Neuroguide software. Results: The results showed that the absolute power of delta $(\mathrm{F}=3.444)$, theta $(\mathrm{F}=51.566)$, alpha $(\mathrm{F}=217.1144)$, and beta $(\mathrm{F}=175.555)$ waves differ between people with depressive disorder and obsessivecompulsive disorder compared to the control group. The delta, theta, and alpha absolute power at frontal lobes of patients with obsessive-compulsion significantly increased, and the alpha and beta absolute power at frontal lobes of patients with major depressive disorder significantly decreased compared to the control group. Conclusions: These results showed that the pattern of brain waves can be posed as an index for diagnosing and follow-upping of the therapeutic outcomes of major depression and obsessive-compulsive disorders Furthermore, it can be used in designing neurofeedback interventions for these disorders.

\section{Keywords:}

1. Obsessive-Compulsive Disorder

2. Electroencephalography

3. Brain Waves

*Corresponding Author: Sajjad Basharpoor

Email: basharpoor_sajjad@uma.ac.ir 


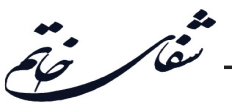

مقايسةُ توان مطلق امواج ناحيةٌ ييشانى مغز در افراد مبتلا به اختلالات افسردكى اساسى، اختلال وسواسى - جبرى و افر اد سالم

\author{
سجاد بشريور "*، شيرين احمدى" يرويز مولوى'، فاضله حيدرى'

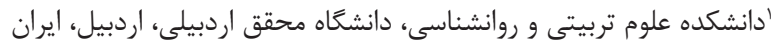

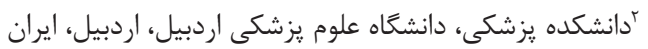

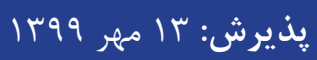

اطلاعات مقاله:

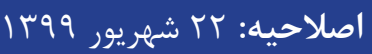

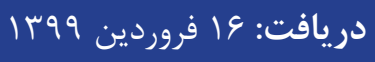

وازمهاى كليدى:

1 - اختلال وسواسى -جبرى

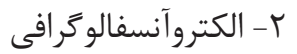
ب- امواج مغزى

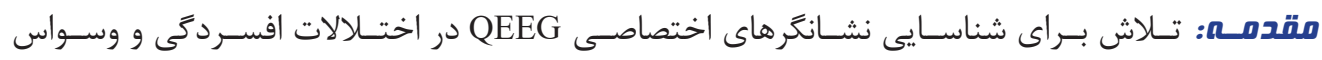

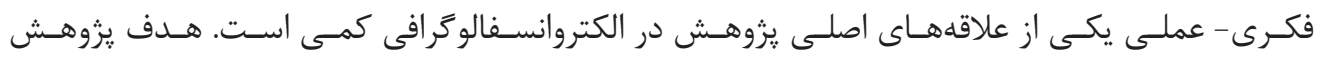

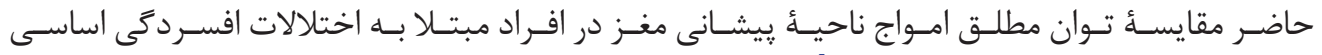

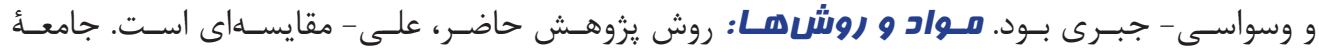

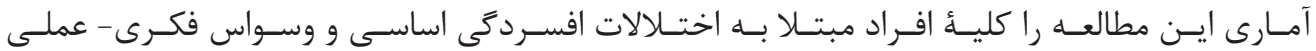

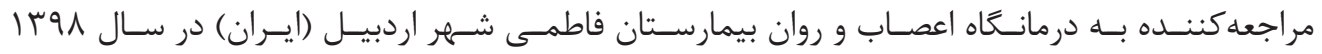

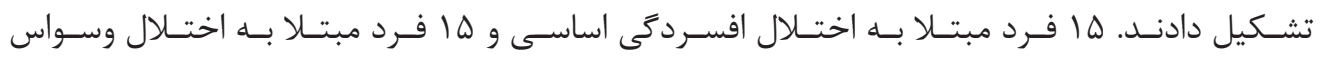

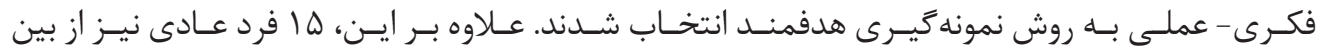

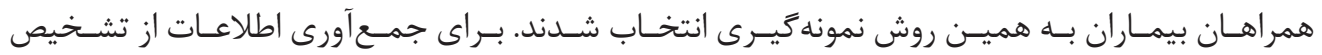

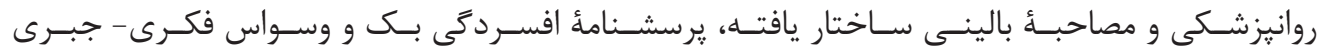

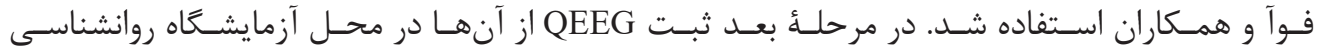

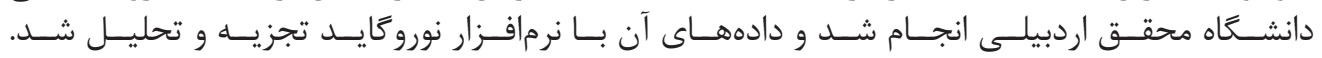

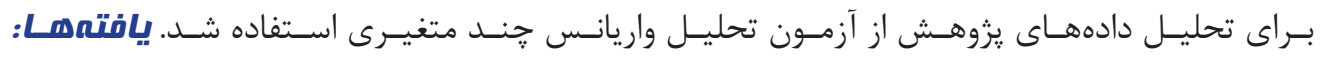

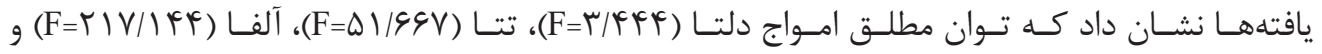

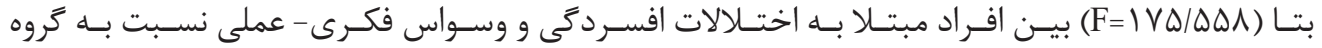

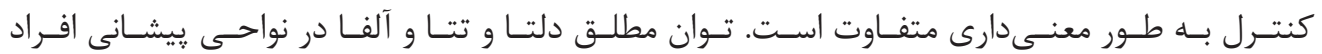

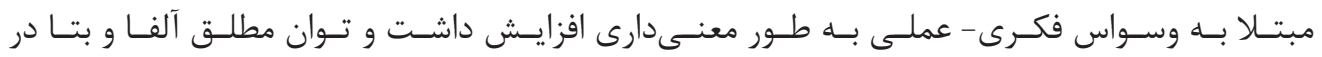

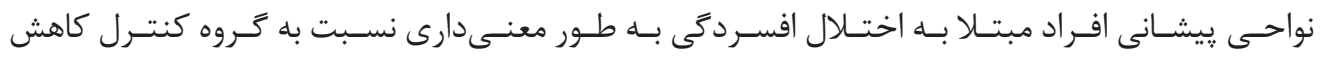

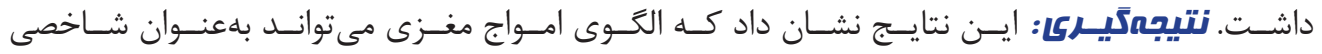

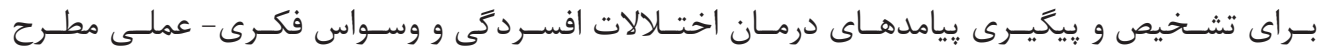

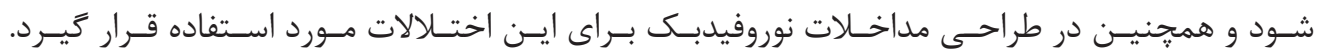




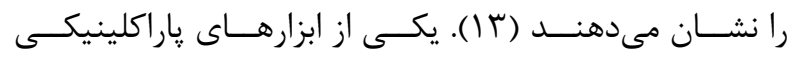

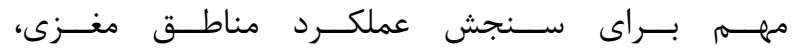

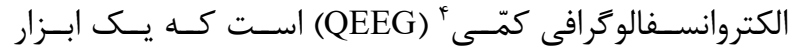

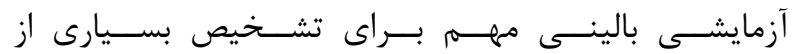

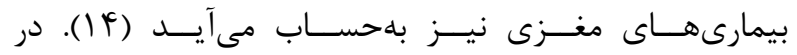

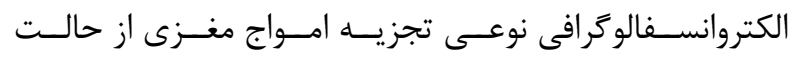

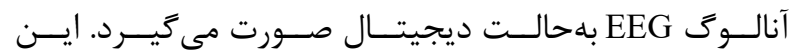

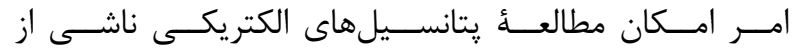

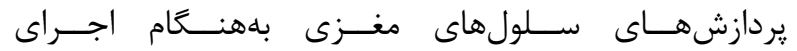

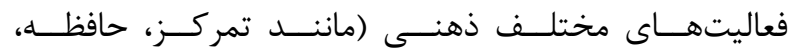

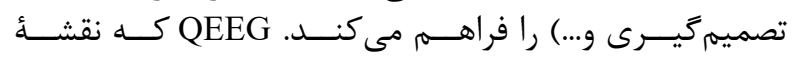

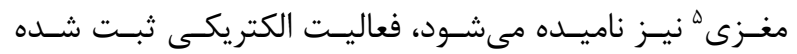

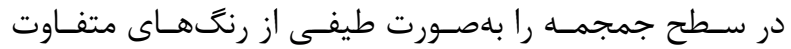

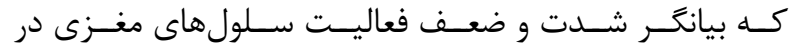

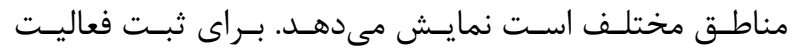

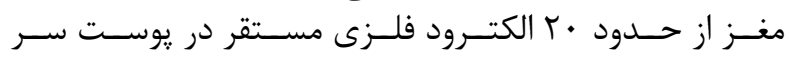

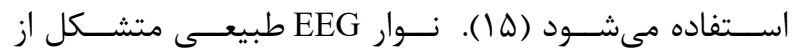

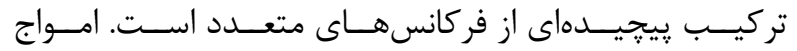

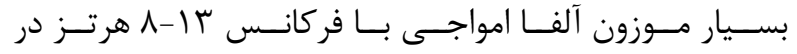

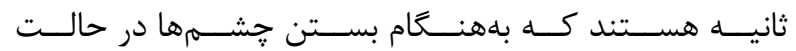

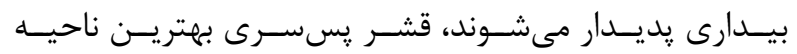

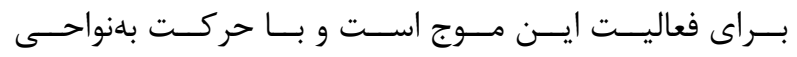

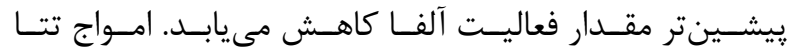

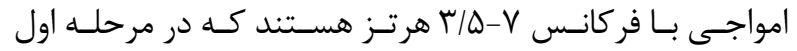

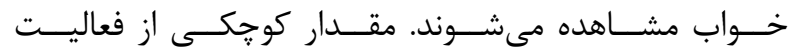

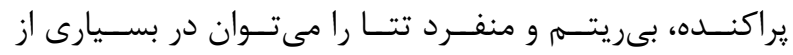
EEG

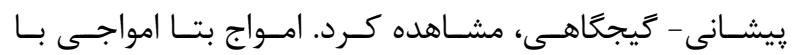

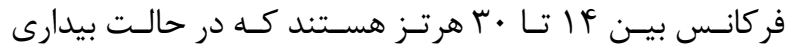

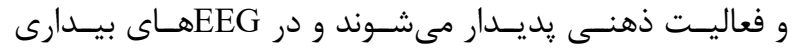

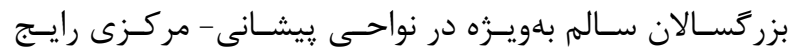

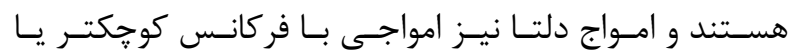

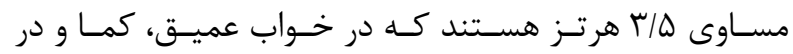

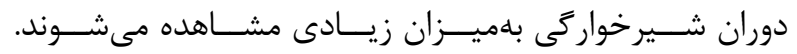

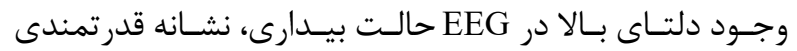

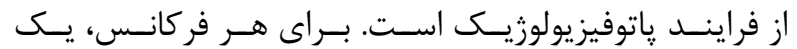

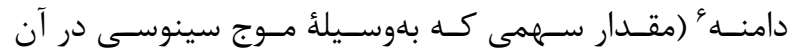

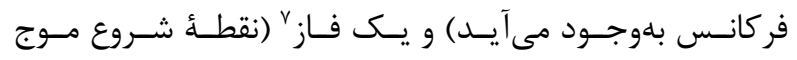

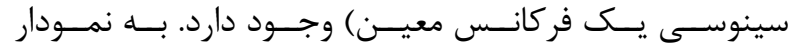

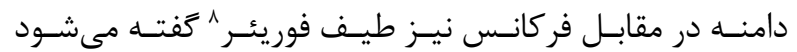

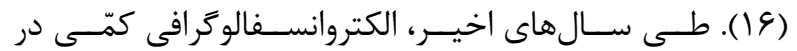

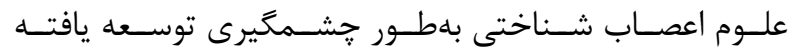

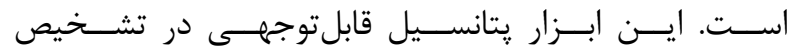

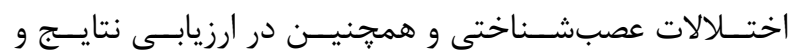

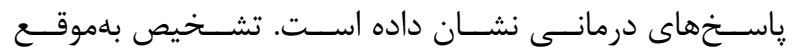

${ }^{1}$ Major Depression Disorder

${ }^{2}$ Sanni, Fm, An, Ayinmode \& Buhari

${ }^{3}$ Obsessiive-Compulsive Disorder

${ }^{4}$ Quantitative Electroencephalogram

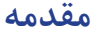

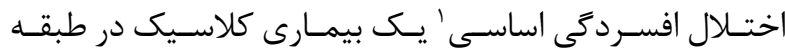

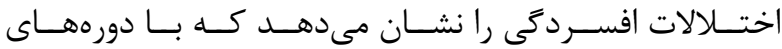

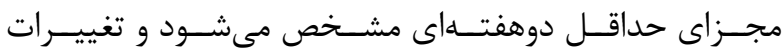

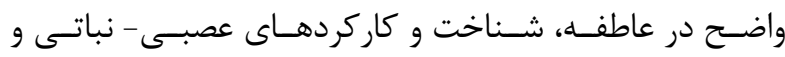

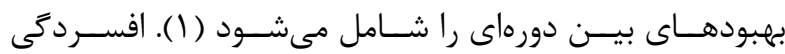

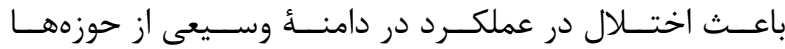

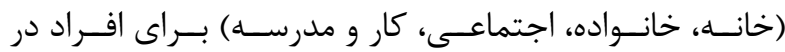

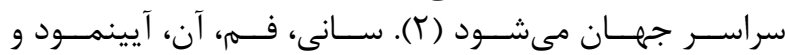

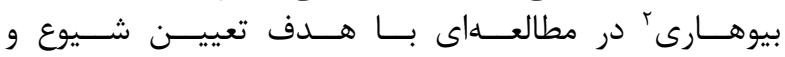

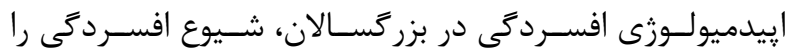

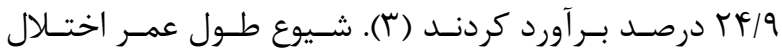

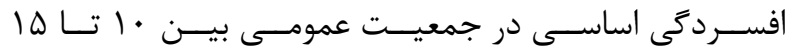

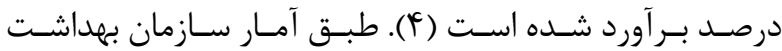

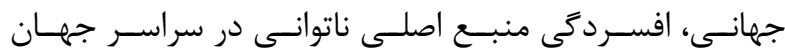

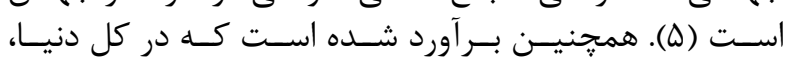

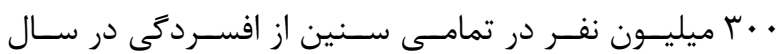

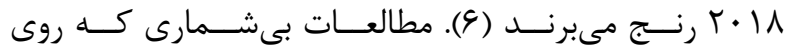

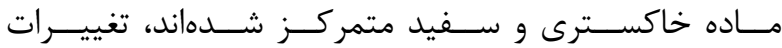

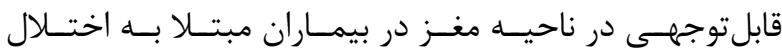

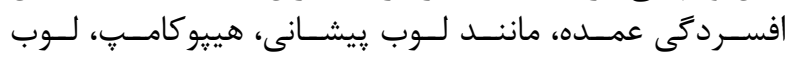

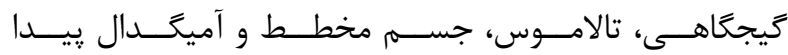

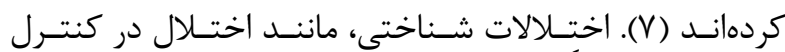

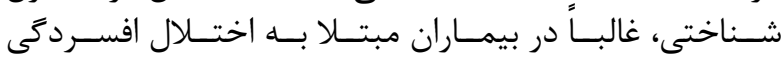

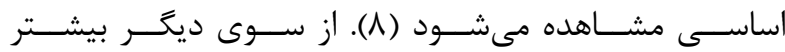

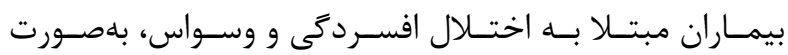

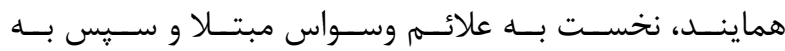

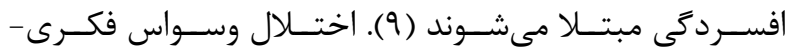

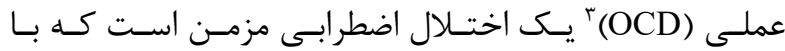

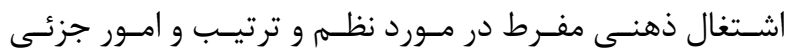

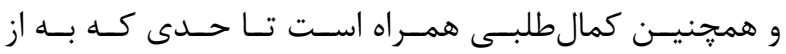

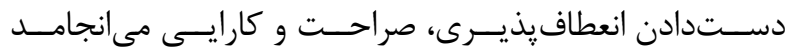

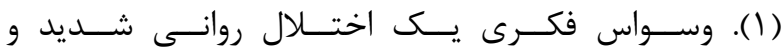

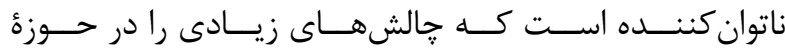

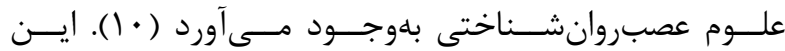

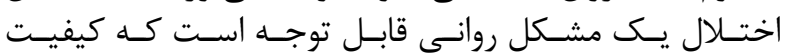

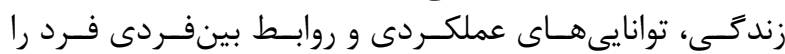

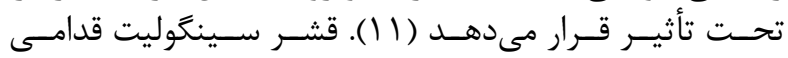

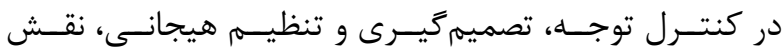

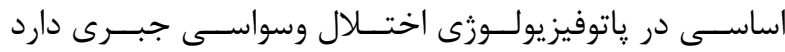

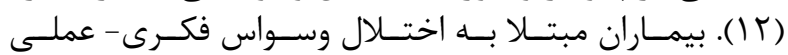

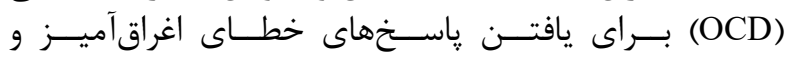

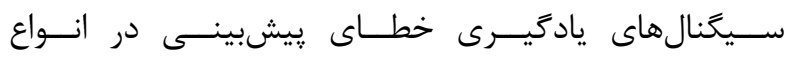

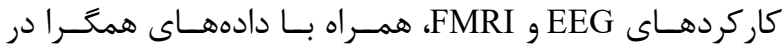

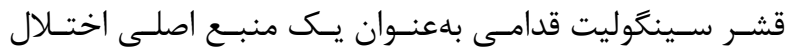

\footnotetext{
${ }^{5}$ Brain Mapping

${ }^{6}$ Amplitude

Phase

${ }^{8}$ Fourier Spectrum
} 


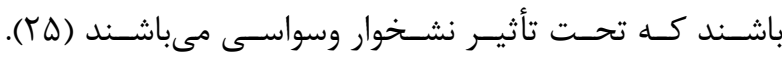

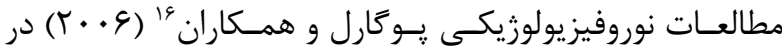

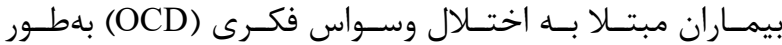

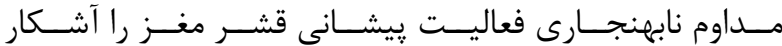

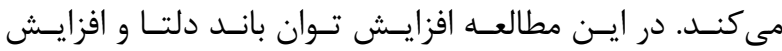

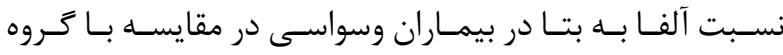

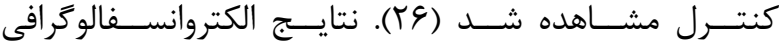

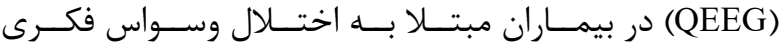

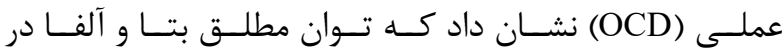

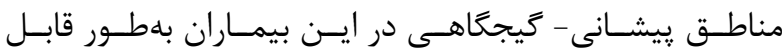

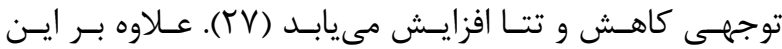

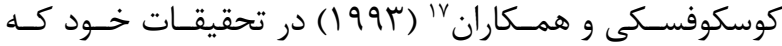

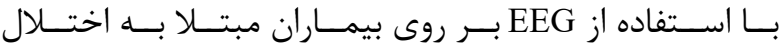

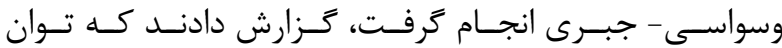

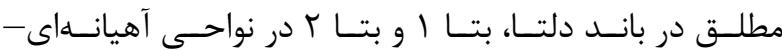

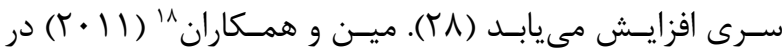

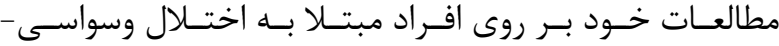

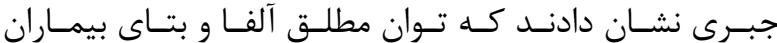

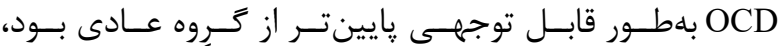

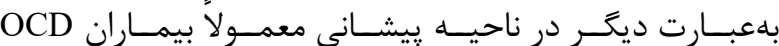

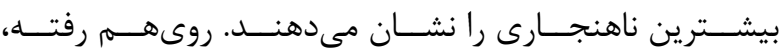

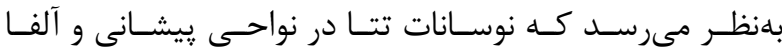

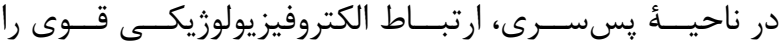

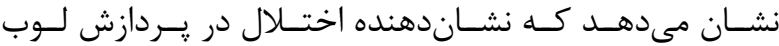

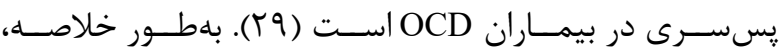

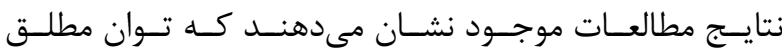

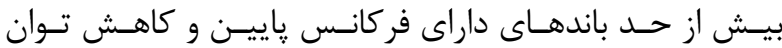

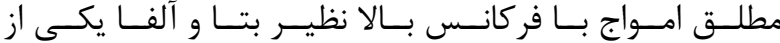

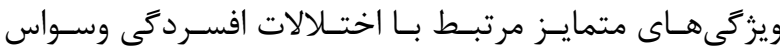

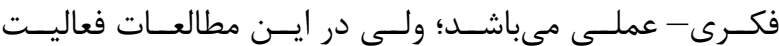

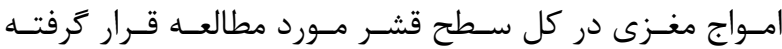

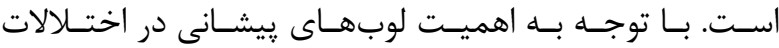

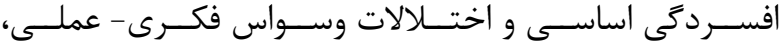

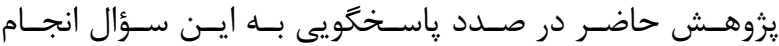

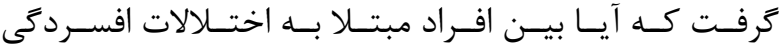

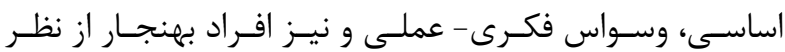

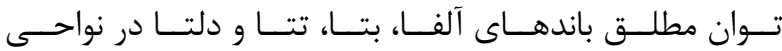

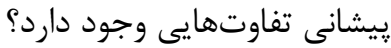

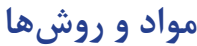

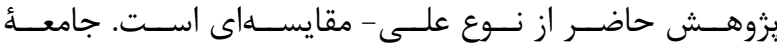

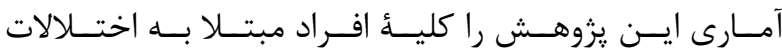

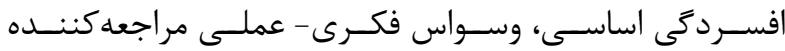

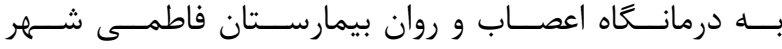

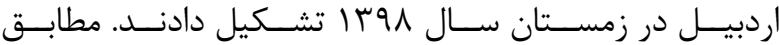

\footnotetext{
${ }^{9}$ Band Oower

${ }^{10}$ Absolute Power or Relative Power

${ }^{11}$ Major Depression

${ }^{12}$ Liu, Zhou, Zhang, Huang

${ }^{13}$ Zoon
}

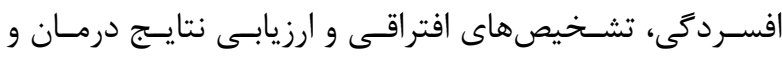

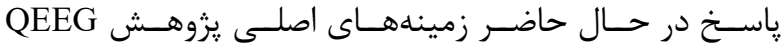

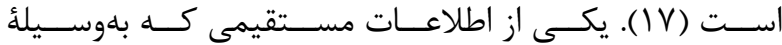

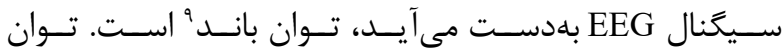

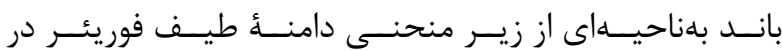

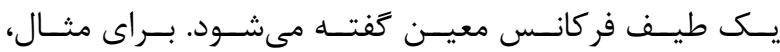

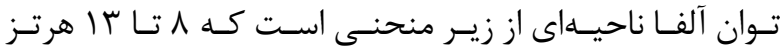

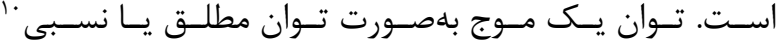

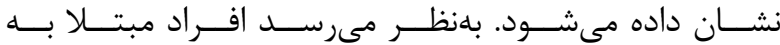

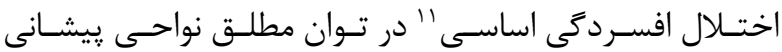

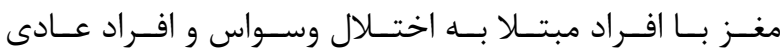

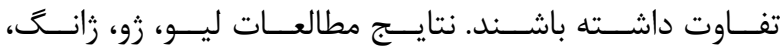

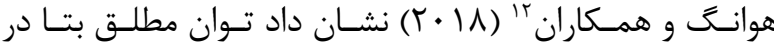

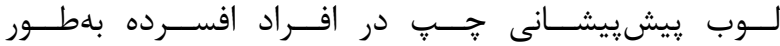

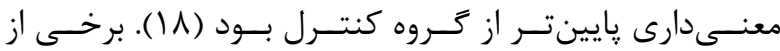

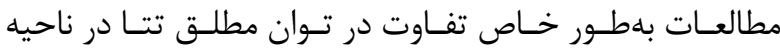

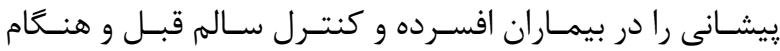

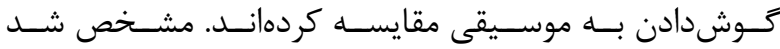

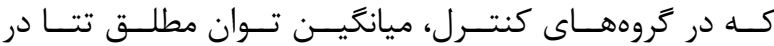

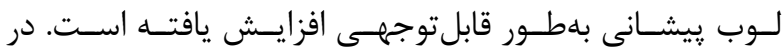

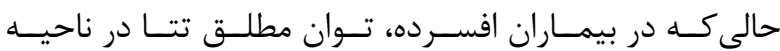

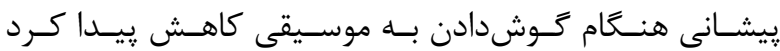

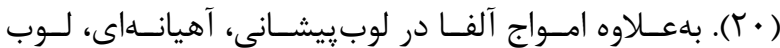

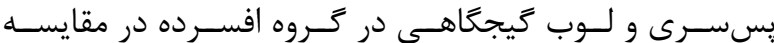

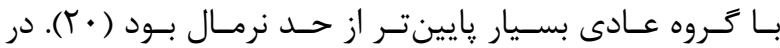

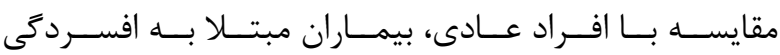

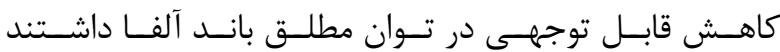

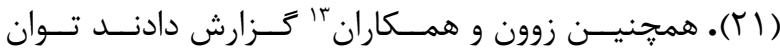

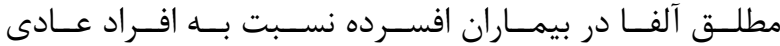

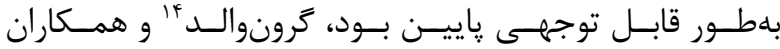

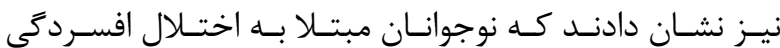

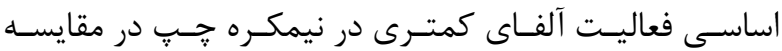

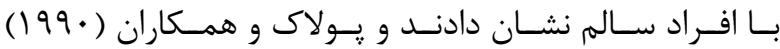

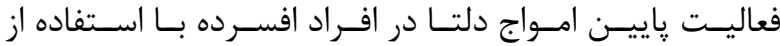

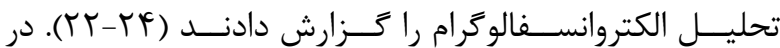

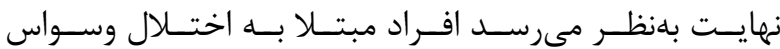

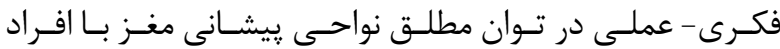

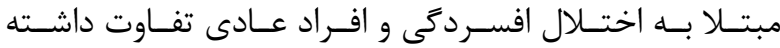

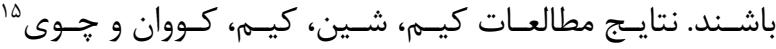

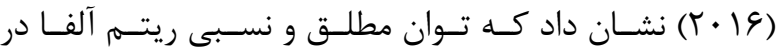

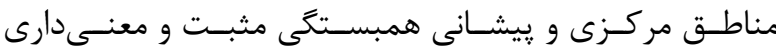

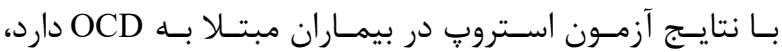

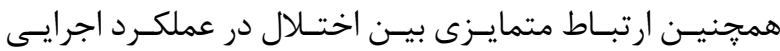

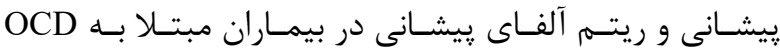

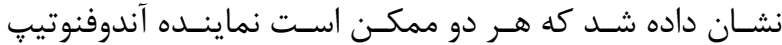

\footnotetext{
${ }^{14}$ Grünewald

${ }^{15} \mathrm{Kim}$, Shin, Kim, Kwon, \& Choi

${ }^{16}$ Pogarell

${ }^{17}$ Kuskowski

${ }^{18}$ Min
} 


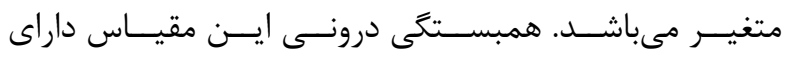

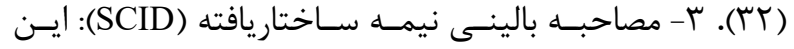

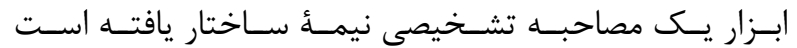

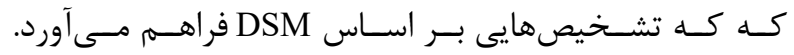
دو نســخئه اصلـى دارد: SCID

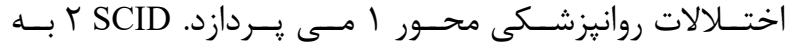

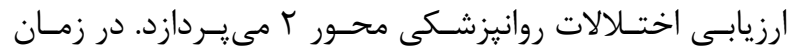

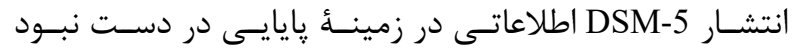

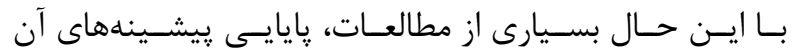

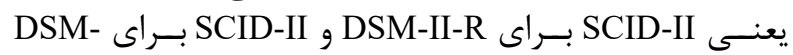

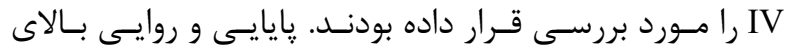

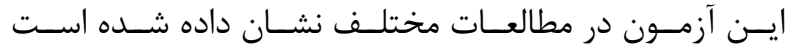
EEG

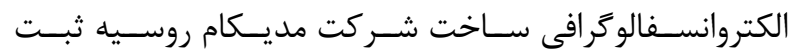

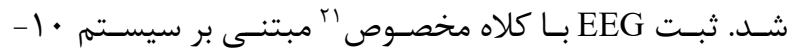

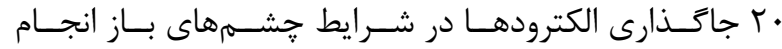

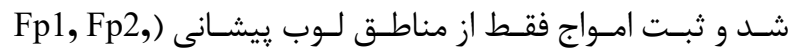

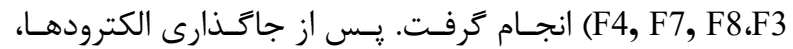

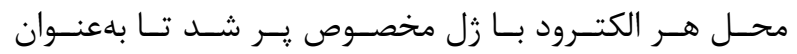

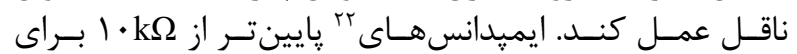

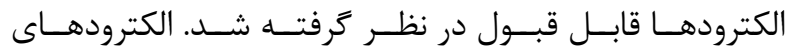

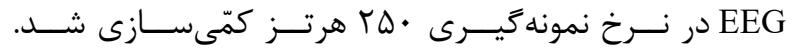

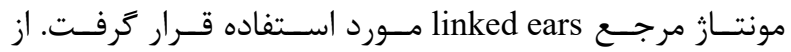

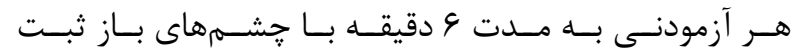

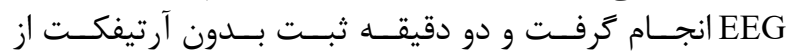

\section{روشهاى تجزيهو تحليل دادهها}

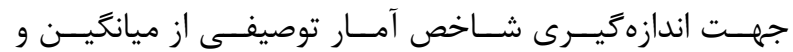

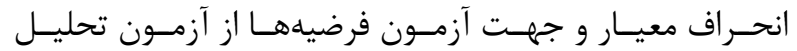

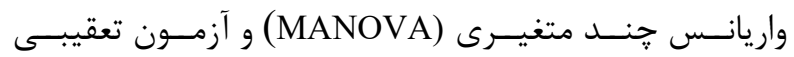

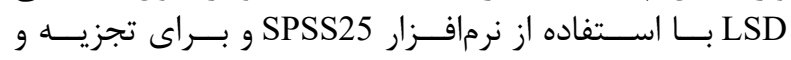

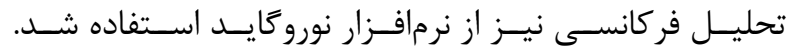

عافتهها

جـدول ا ميانكيـن و انحراف معيار زروههاى آزمايشـى (اختلال

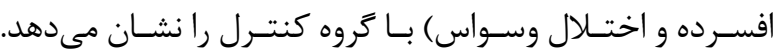

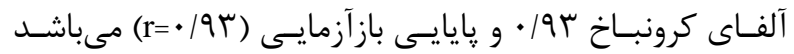

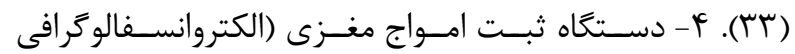
EEG

آمــار اخــذ شـده از بيمارسـتان، ايــن تعـداد بــراى بيمــاران

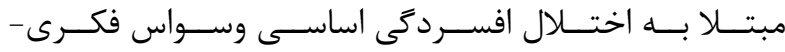

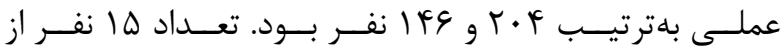

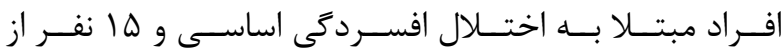

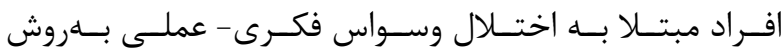

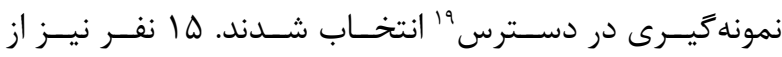

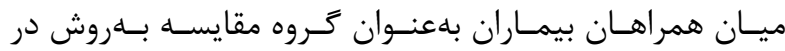

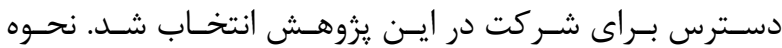

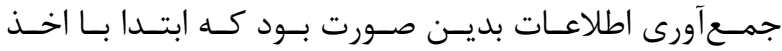

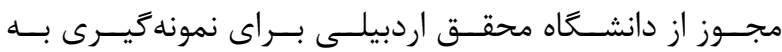

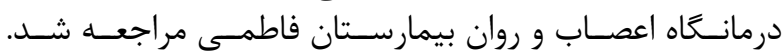

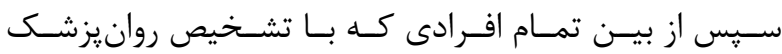

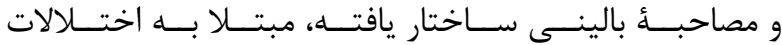

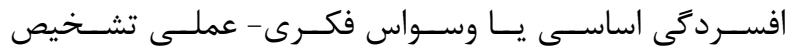

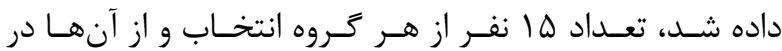

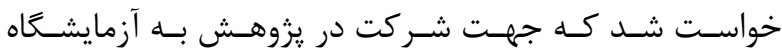

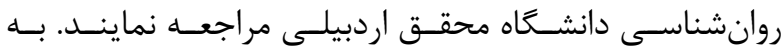

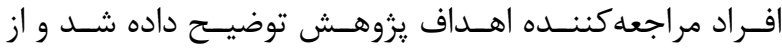

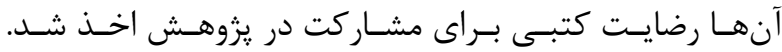

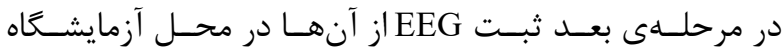

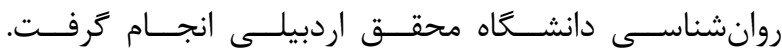

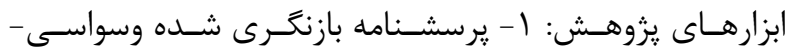

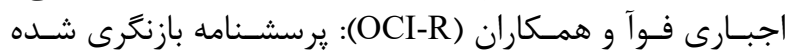

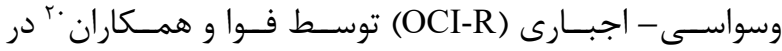

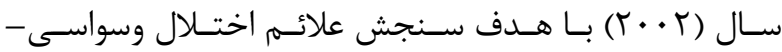

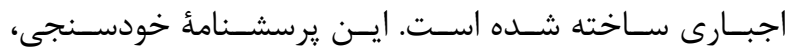

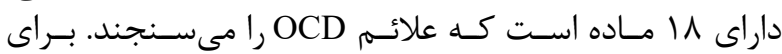

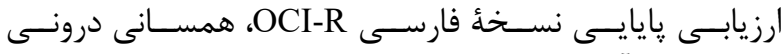

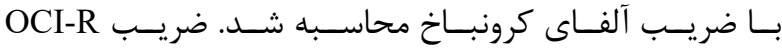

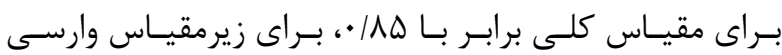

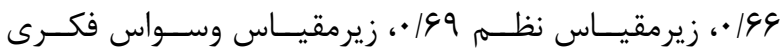

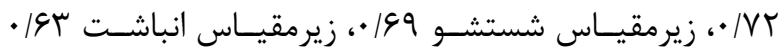

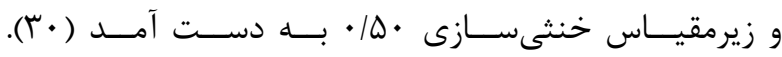

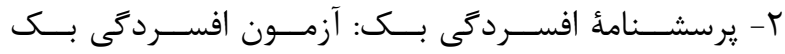

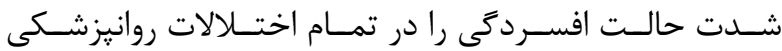

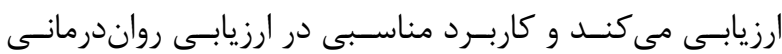

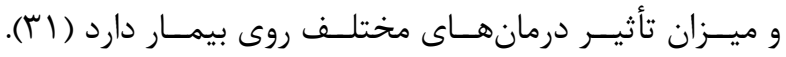

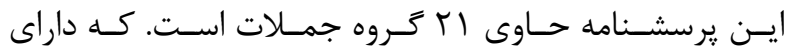

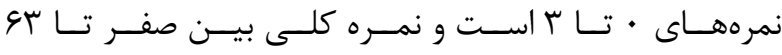
جدول ا- ميانكَين و انحراف معيار سن كروههاى آزمايشى و كنترل

\begin{tabular}{|c|c|c|c|c|}
\hline انحراف معيار & ميانگين & فراوانى & تروه & متغير \\
\hline IT/TK & $\mathrm{r} \omega / \cdot \mathrm{V}$ & 10 & اختلال افسرده & \multirow{3}{*}{ سن } \\
\hline$|\omega| \cdot \Lambda$ & re/gV & 10 & اختلال وسواس & \\
\hline IT/AV & rV/AV & 10 & كنترل & \\
\hline
\end{tabular}

${ }^{19}$ Convenience Sampling

${ }^{20} \mathrm{Foa}$

${ }^{22}$ Electrode impedances

${ }^{23}$ Neuroguide 


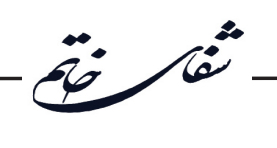

جدول r- آمارههاى توصيفى مربوط به توان مطلق امواج دلتا، تتا، آلفاو بتا در مناطق بيشانى

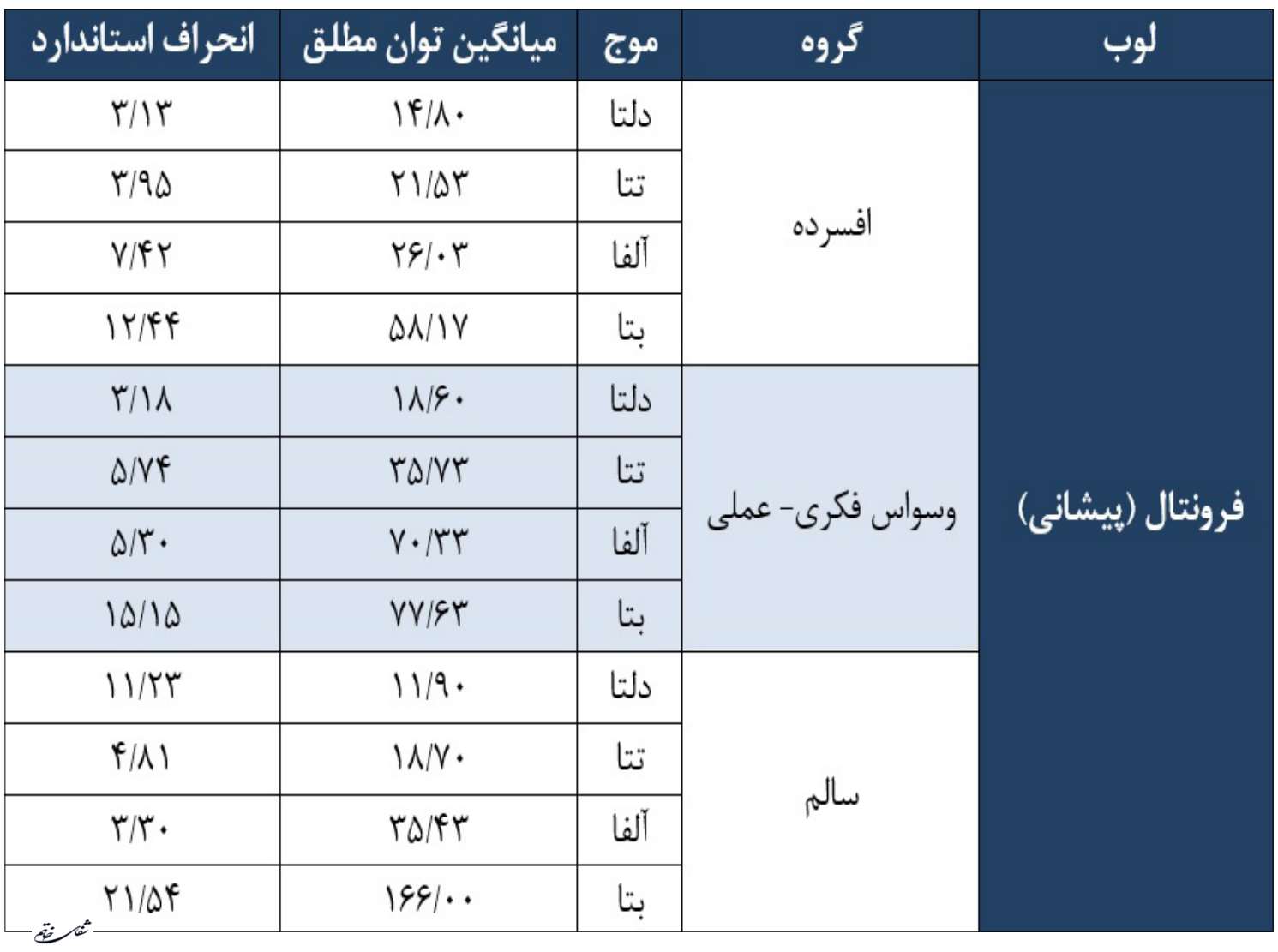

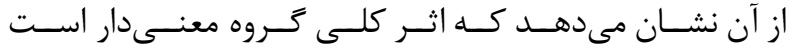

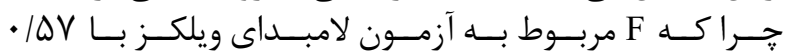

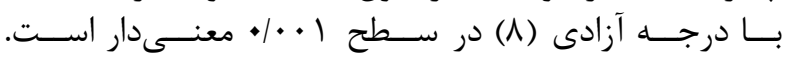

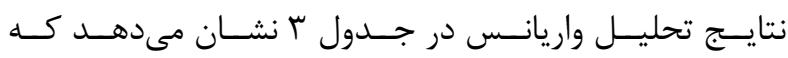

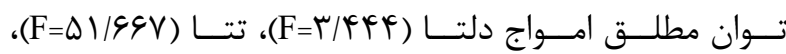

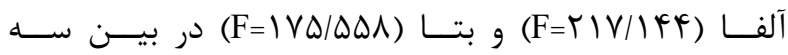

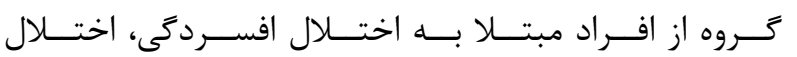

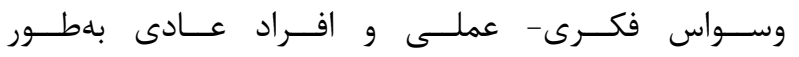

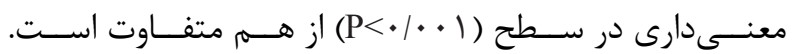

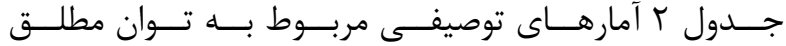

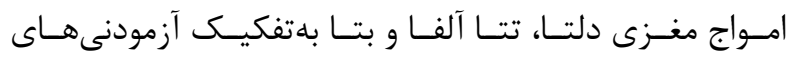

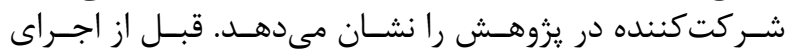

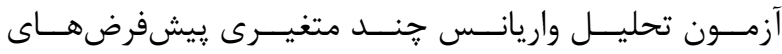

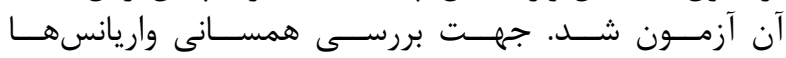

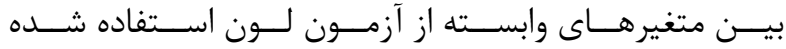

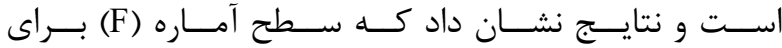

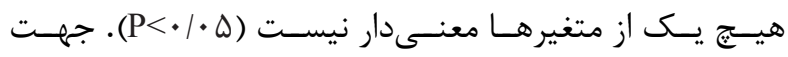

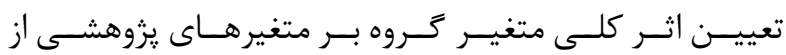

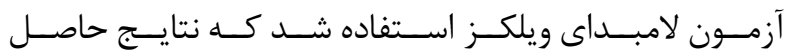

جدول بـ- نتايج آزمون تحليل واريانس קهندمتغيرى (مانوا) براى فعاليت امواج (دلتا، تتا، آلفا و بتا) در مناطق بيشانى

\begin{tabular}{|c|c|c|c|c|c|c|c|}
\hline Eta Squared & P-value & $\mathbf{F}$ & MS & Df & SS & متغير وابسته & منبع \\
\hline.$/ 14$ & $\cdot 1 \cdot 4$ & T/ppp & 19N/9T. & $r$ & rrv|Agl & دلتا & \multirow{4}{*}{ تروه } \\
\hline$\cdot|V|$ & $\cdot 1 \cdot \cdot 1$ & $01 / 99 \mathrm{~V}$ & سF & t & TYAN/GG & تتا & \\
\hline .191 & $.1 \cdot \cdot 1$ & $r I V / I F F$ & $\Lambda I V \cdot / V \cdot \Delta$ & $r$ & $|\varepsilon r| q|/ F|$. & آلفا & \\
\hline .119 & $\cdot 1 \cdot \cdot 1$ & $\mid V \Delta / \Delta \Delta \Lambda$ & FqGYI/VYI & $r$ & QqTFT/FFT & بتا & \\
\hline
\end{tabular}




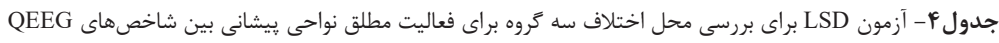

\begin{tabular}{|c|c|c|c|c|c|}
\hline معنى دارى & خطاى انتحراف استاندارد & تفاوت ميانجين & كروما & \multicolumn{2}{|c|}{ وايسته متَغير } \\
\hline $.11 \mathrm{Fs}$ & $r / \Delta \Delta V F q$ & -r/VQY. & وسواس & \multirow{2}{*}{ اقسرده } & \multirow{6}{*}{ دلتا } \\
\hline - MAY & $r / \Delta \Delta V F q$ & $r / 9 . .$. & سالهم & & \\
\hline .1149 & T/DQVFq & r/VAr. & افسرده & \multirow{2}{*}{ وسواس } & \\
\hline$+1 \cdot 1$ & $Y / \Delta \Delta V F q$ & 9/994. & سالم & & \\
\hline ./TSY & $r / \Delta \Delta V F q$ & $-r / 9 \ldots$ & اقسرده & \multirow{2}{*}{ سالهم } & \\
\hline$+1+1$ & Y/DQVFq & $-9 / 994 .=$ & وسواس & & \\
\hline$* 1 * 1$ & I/V9IVS & $-|f /| V \gamma+=$ & وسواس & \multirow{2}{*}{ افسرده } & \multirow{6}{*}{ تتا } \\
\hline . MTF & I/VqIVs & r(A)FY & سـالم & & \\
\hline$* 1+\cdots 1$ & I/V9IVS & $|f /| \gamma \gamma+=$ & افسرده & \multirow{2}{*}{ وت بوسواس } & \\
\hline$\cdot 1 \cdots 1$ & I/V9IVs & $19 / 991 Y^{-2=}$ & سـالم & & \\
\hline . IITF & I/V9IVs & $-Y(\Lambda) F Y$ & اقفرده & \multirow{2}{*}{ سالهم } & \\
\hline$\cdot 1+\cdots 1$ & I/VqIVS & $-19 / 991 Y^{2 \pi}$ & وسواس & & \\
\hline$+1+\infty)$ & T/YTQA & $-F F / Y \cdot G \cdot \pi=$ & ووسواس & \multirow{2}{*}{ افسرده } & \multirow{6}{*}{ آلثا } \\
\hline$+1 \cdots 1$ & T/YYQA & $-9 / 4 Y \ldots=$ & 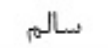 & & \\
\hline$+1+\cdots)$ & T/YYQA & FF/Y.9.AI & اقسرده & \multirow{2}{*}{ وت وسواس } & \\
\hline$+1 \cdots 1$ & T/YYQA & YFIAVG. $=$ & 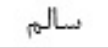 & & \\
\hline$+1+\cdots)$ & T/YTQAN & $9 / 4+\ldots=$ & افسرده & \multirow{2}{*}{ ساله } & \\
\hline$+1 * 1$ & T/YTQAN & - YFIAVG. ${ }^{2 \pi}$ & وسواس & & \\
\hline$* 1+r$ & 9/1 ҮА१९ & $-19 / F Y \cdot V^{2 x}$ & ووسواس & \multirow{2}{*}{ اقسرده } & \multirow{6}{*}{ يتا } \\
\hline$\cdot 1+\cdots 1$ & ร/イ^१९ & $-1+V / 9+1 Y^{2=}$ & سالم & & \\
\hline$\cdot 1 \cdots Y$ & S/ & $19 / F r \cdot V^{2 x}$ & افسرده & \multirow{2}{*}{ وسواس } & \\
\hline$+1+\cdots)$ & 9/1 ҮА१я & $-\Lambda N F \Lambda \cdot V^{2 x}$ & 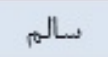 & & \\
\hline$+1+\cdots)$ & 9/1 ҮА१९ & $1+\gamma / 9+1 r^{22}$ & اقسرده & \multirow{2}{*}{ سالهم } & \\
\hline$+1+\cdots 1$ & 9/ & $N N F A \cdot V^{2=}$ & وسواس & & \\
\hline
\end{tabular}

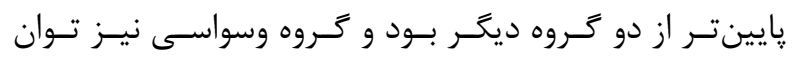

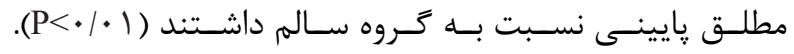
بحث و نتيجه كيرى

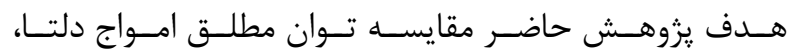

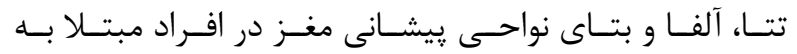

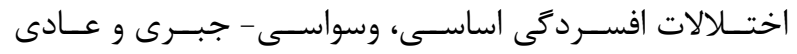

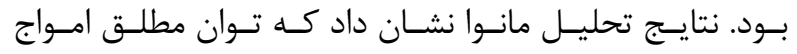

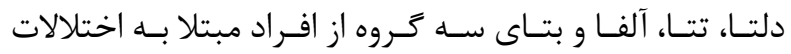

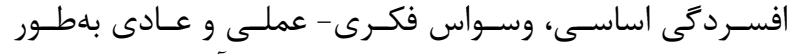

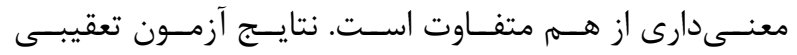

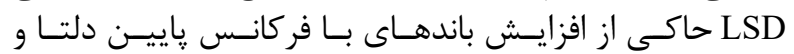

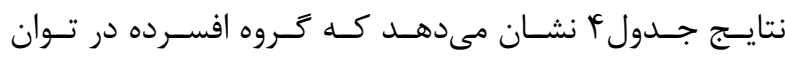

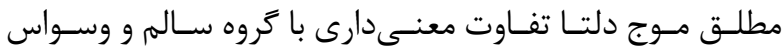

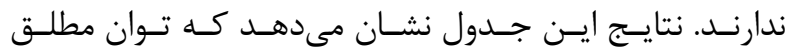

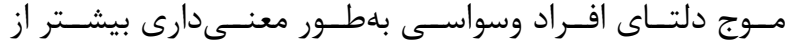

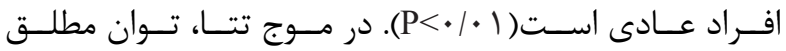

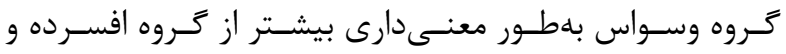

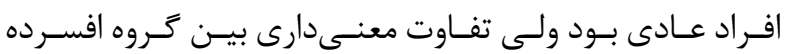

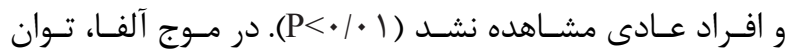

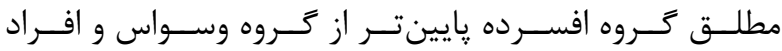

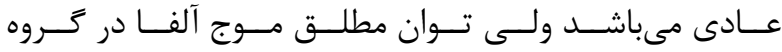

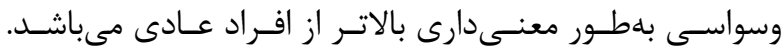

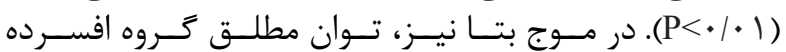




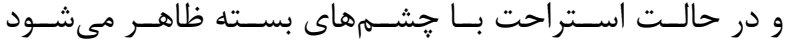

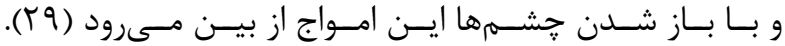

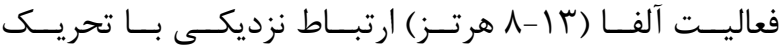

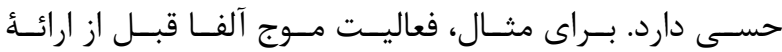

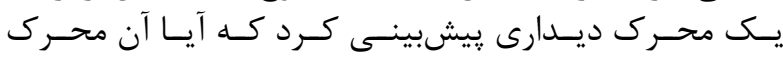

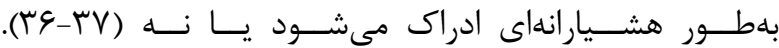

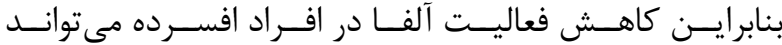

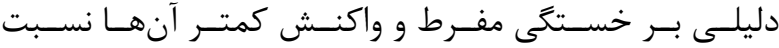

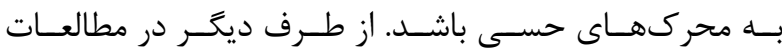

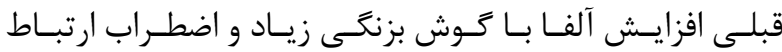

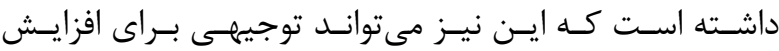

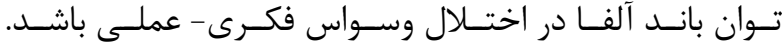

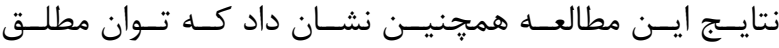

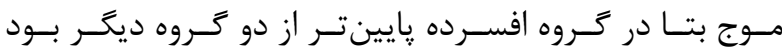

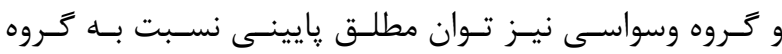

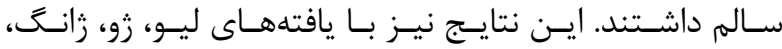

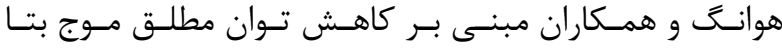

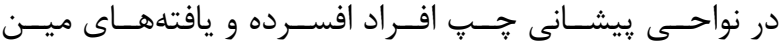

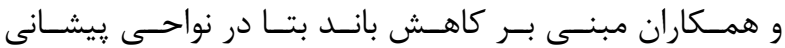

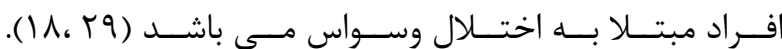

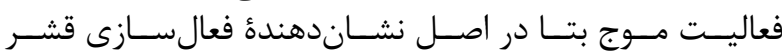

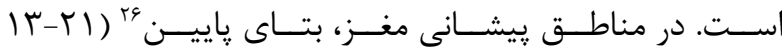

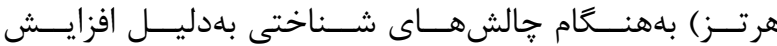

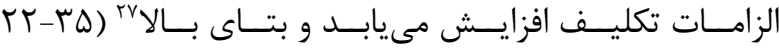

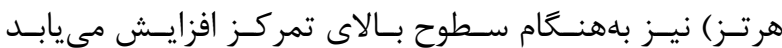

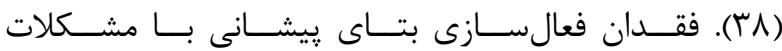

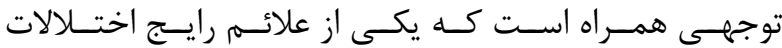

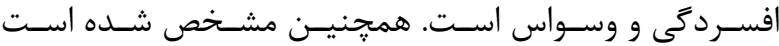

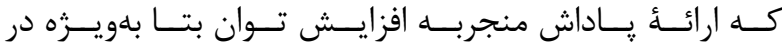

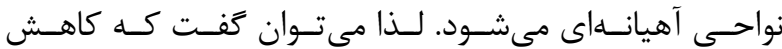

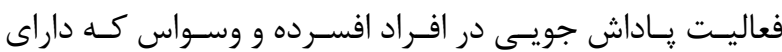

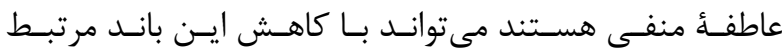

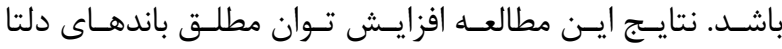

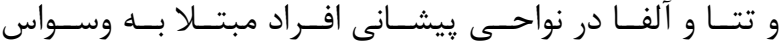

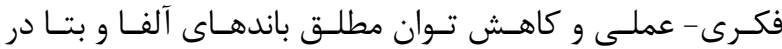

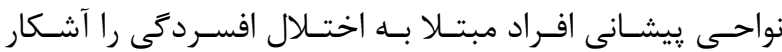

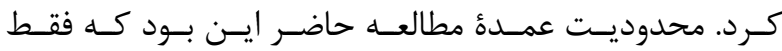

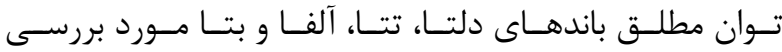

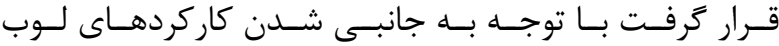

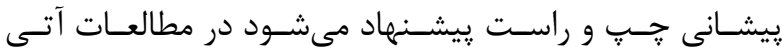

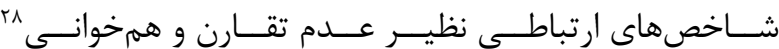

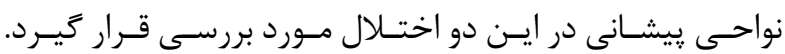

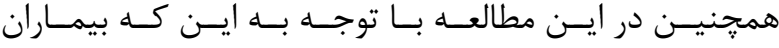

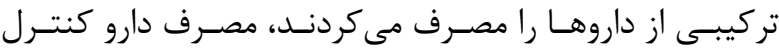

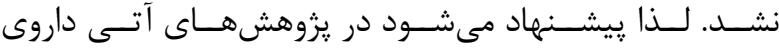

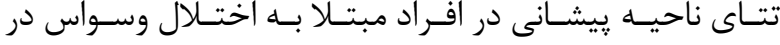

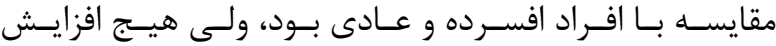

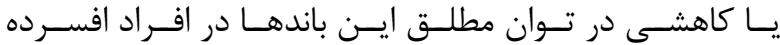

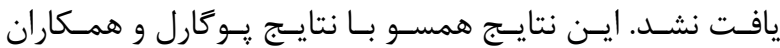

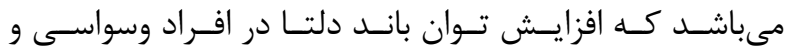

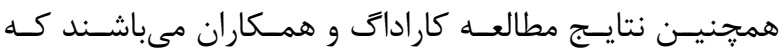

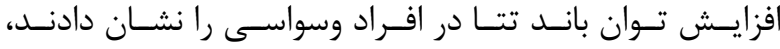

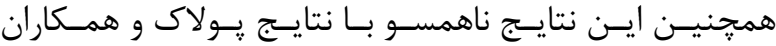

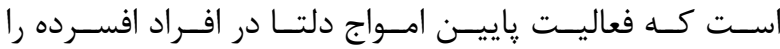

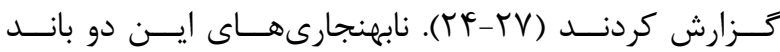

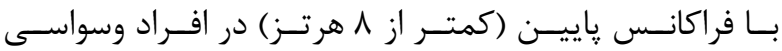

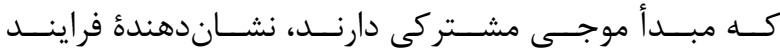

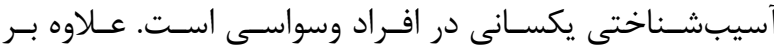

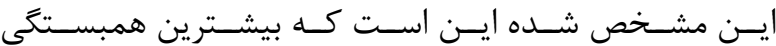

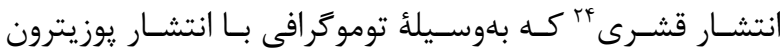

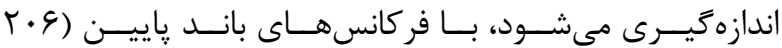

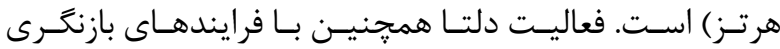

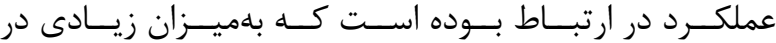

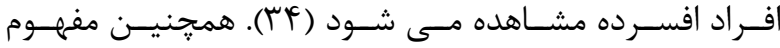

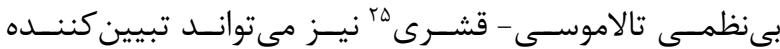

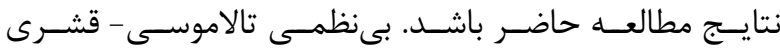

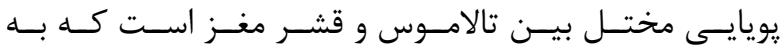

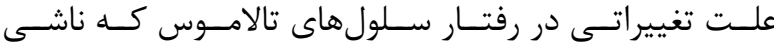

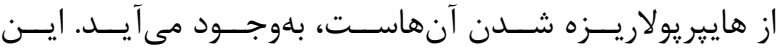

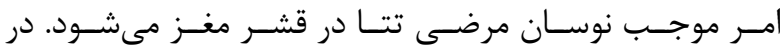

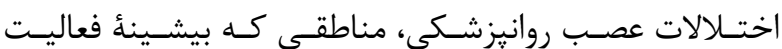

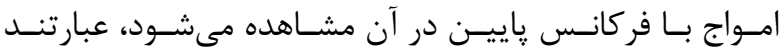

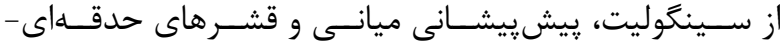

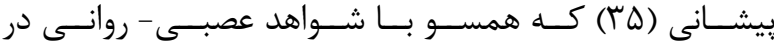

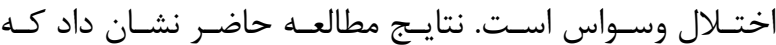

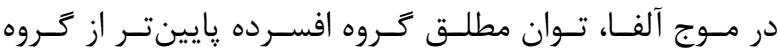

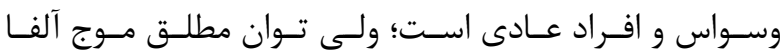

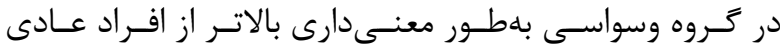

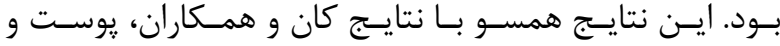

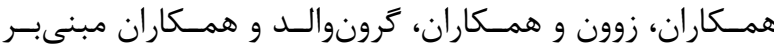

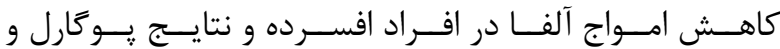

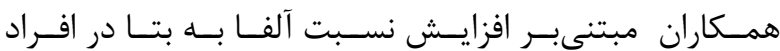

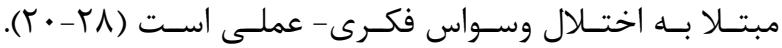

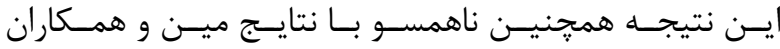

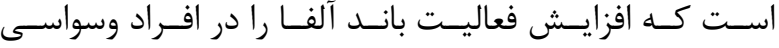

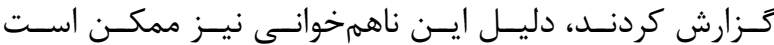

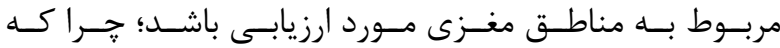

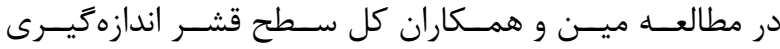

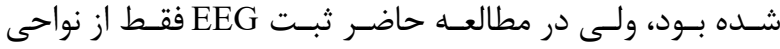

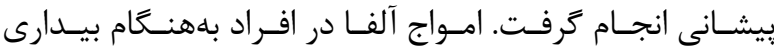




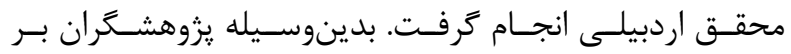

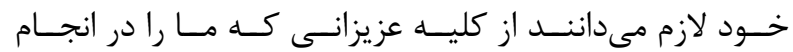

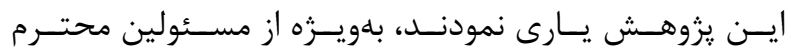

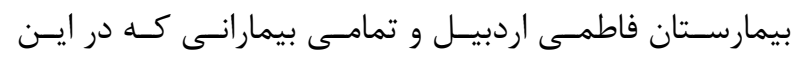
يزوهـش شـركت كردنـد، صميمانـهـ قدردانسى و تشـكر نمايند.

1. Association AP. Diagnostic and statistical manual of mental disorders (DSM5®): American Psychiatric Pub; 2013.

2. Greer TL, Joseph JK. Pharmacological and Nonpharmacological Treatment Effects on Functional Outcomes in Major Depressive Disorder. Major Depressive Disorder: Elsevier; 2020. p. 131-146.

3. Sanni O, Fm O, An A, Ayinmode B, Buhari O. Epidemiology of Depression in a Primary Care Setting in North Central Nigeria. Age (years). 2020; 20(29): 32.

4. Lépine J-P, Briley M. The increasing burden of depression. Neuropsychiatric disease and treatment. 2011; 7(Suppl 1): 3 .

5. Depression and other common mental disorders: Global health estimates [Internet]. 2017.

6. Key facts about depression 2018 Geneva: World Health Organization [Internet]. 2018.

7. Zhang FF, Peng W, Sweeney JA, Jia ZY, Gong QY. Brain structure alterations in depression: psychoradiological evidence. CNS neuroscience \& therapeutics. 2018; 24(11): 994-1003.

8. Jiao $\mathrm{K}$, Xu H, Teng $\mathrm{C}$, Song $\mathrm{X}$, Xiao C, Fox PT, et al. Connectivity patterns of cognitive control network in first episode medicationnaive depression and remitted depression. Behavioural brain research. 2020; 379: 112381.

9. Rasmussen SA, Tsuang MT. Clinical characteristics and family history in DSM-III obsessive-compulsive disorder. The American journal of psychiatry. 1986.

10. Robbins TW, Vaghi MM, Banca P. Obsessive-compulsive disorder: puzzles and prospects. Neuron. 2019; 102(1): 27-47.

11. Taube-Schiff M, Rector NA, Young R, Larkin P, Richter MA. Filling the gap for obsessive-compulsive disorder services in Canada: Implementing an intensive care program. The Journal of Nervous and Mental Disease. 2020; 208(1): 38-47.

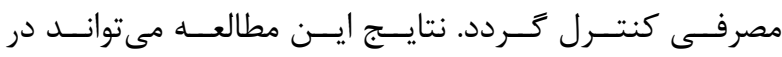

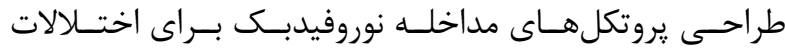

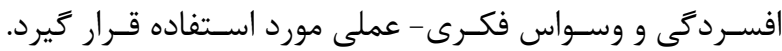
تشكر و قدردانى

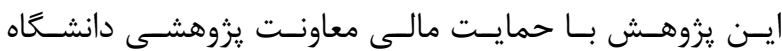
منابع

12. Novak C, Swain H, McLean B, Tran C, Vitale A, Witkemper $\mathrm{K}$, et al. F25. Increased Adverse Childhood Experiences Predict Worse Acute Pain and Psychological Symptoms After Sexual Assault. Biological Psychiatry. 2019; 85(10): S222.

13. Murray GK, Knolle F, Ersche KD, Craig KJ, Abbott S, Shabbir SS, et al. Dopaminergic drug treatment remediates exaggerated cingulate prediction error responses in obsessive-compulsive disorder. Psychopharmacology. 2019; 236(8): 2325-336.

14. Tabanfar Z, Firoozabadi S, Khodakarami Z, Shankayi Z. Analysis of Electroencephalogram Data during Rest in Patients with Brain Tumor. Modares Journal of Biotechnology. 2018; 9(4): 653-58.

15. Basharpoor S, Heidari F, Molavi P. EEG coherence in theta, alpha, and beta bands in frontal regions and executive functions. Applied Neuropsychology: Adult. 2019: 1-8.

16. Banich MT, Compton RJ. Cognitive Neuroscience. 2011. Translated by Bashirpoor S, Isa Zadegan A, Ardabil: University of Mohaghegh Ardebil University Press 2017.

17. Yadollahpour A, Nasrollahi H. Quantitative electroencephalography for objective and differential diagnosis of depression: a comprehensive review. Global journal of health science. 2016;8(11):249-256.

18. Liu L, Zhou H, Zhang M, Huang J, Feng L, Zhong N, editors. Resting EEG Features and Their Application in Depressive Disorders. 2018 IEEE/WIC/ACM International Conference on Web Intelligence (WI); 2018: IEEE.

19. Dharmadhikari A, Tandle A, Jaiswal S, Sawant V, Vahia V, Jog N. Frontal theta asymmetry as a biomarker of depression. East Asian Archives of Psychiatry. 2018;28(1):17.

20. Kan D, Lee P, editors. Decrease alpha waves in depression: An electroencephalogram (EEG) study. 2015 International Conference on BioSignal Analysis, Processing and Systems (ICBAPS); 2015: IEEE.

21. Post JM. A Brotherhood of Tyrants: Manic 
Depression and Absolute Power. LWW; 1996. p. 647-48.

22. Zoon HF, Veth C, Arns M, Drinkenburg W, Talloen W, Peeters PJ, et al. EEG alpha power as an intermediate measure between brain-derived neurotrophic factor Val66Met and depression severity in patients with major depressive disorder. Journal of Clinical Neurophysiology. 2013; 30(3): 261-67.

23. Grünewald BD, Greimel E, Trinkl M, Bartling J, Großheinrich N, Schulte-Körne G. Resting frontal EEG asymmetry patterns in adolescents with and without major depression. Biological psychology. 2018; 132: 212-16.

24. Pollock VE, Schneider LS. Quantitative, waking EEG research on depression. Biological Psychiatry. 1990; 27(7): 757-80.

25. Kim S, Shin JE, Kim MJ, Kwon JS, Choi SH. Correlation between quantitative electroencephalogram findings and neurocognitive functions in patients with obsessive-compulsive disorder and schizophrenia. Korean J Biol Psychiatry. 2016; 23(4): 193.

26. Pogarell O, Juckel G, Mavrogiorgou P, Mulert C, Folkerts M, Hauke W, et al. Symptomspecific EEG power correlations in patients with obsessive-compulsive disorder. International journal of psychophysiology. 2006; 62(1): 87-92.

27. Karadag F, Oguzhanoglu NK, KURT T, Oguzhanoglu A, Atesci F, ÖZDEL O. Quantitative EEG analysis in obsessive compulsive disorder. International journal of neuroscience. 2003;113(6):833-47.

28. Kuskowski MA, Malone SM, Kim SW, Dysken MW, Okaya AJ, Christensen KJ. Quantitative EEG in obsessive-compulsive disorder. Biological psychiatry. 1993; 33(6): 423-30.

29. Min B-K, Kim SJ, Park JY, Park H-J. Prestimulus top-down reflection of obsessive-compulsive disorder in EEG frontal theta and occipital alpha oscillations. Neuroscience letters. 2011;496(3):181-185.

30. Foa EB, Huppert JD, Leiberg S, Langner R, Kichic R, Hajcak G, et al. The Obsessive-Compulsive Inventory: development and validation of a short version. Psychological assessment. 2002; 14(4): 485.

31. Beck AT, Steer RA, Brown GK. Beck depression inventory-II. San Antonio. 1996; 78(2): 490-98.

32.Dabson KS, P. M. Psychometrics characteristic of beck depression inventory-ii in a big sample of major deprssive patients. J Rehabil. 2007; 8(8): 8-82.

33. First MB, Spitzer RL, Giben M, JBW. W. Semistructred by cilinical interview for disorder of DSM-IV-TR. Tehran: danzhe; 2010.

34. Yordanova J, Falkenstein M, Hohnsbein J, Kolev V. Parallel systems of error processing in the brain. Neuroimage. 2004; 22(2): 590-602.

35. Llinás RR, Ribary U, Jeanmonod D, Kronberg E, Mitra PP. Thalamocortical dysrhythmia: a neurological and neuropsychiatric syndrome characterized by magnetoencephalography. Proceedings of the National Academy of Sciences. 1999; 96(26): 15222-227.

36. Hanslmayr S, Aslan A, Staudigl T, Klimesch W, Herrmann CS, Bäuml K-H. Prestimulus oscillations predict visual perception performance between and within subjects. Neuroimage. 2007; 37(4): 1465-473.

37. Kranczioch C, Debener S, Maye A, Engel AK. Temporal dynamics of access to consciousness in the attentional blink. Neuroimage. 2007; 37(3): 947-955.

38. Basharpoor S, Amani S, Rashid S. The Relationship Between QEEG Waves as a Neurophysiological Markers in FP1 / Fp2 Areas and Multiple Aspects of Impulsivity, International Journal of High Risk Behaviors and Addiction. 2018; 7(3): e58637. 